Bull. Austral. Math. Soc.

$54 \mathrm{c} 30,54 \mathrm{C} 25$

VOL. 68 (2003) [471-480]

\title{
ON BASIC EMBEDDINGS OF COMPACTA INTO THE PLANE
}

\author{
Neža Mramor-Kosta and Eva TrenKLerová
}

A compactum $K \subset \mathbb{R}^{2}$ is said to be basically embedded in $\mathbb{R}^{2}$ if for each continuous function $f: K \rightarrow \mathbb{R}$ there exist continuous functions $g, h: \mathbb{R} \rightarrow \mathbb{R}$ such that $f(x, y)$ $=g(x)+h(y)$ for each point $(x, y) \in K$. Sternfeld gave a topological characterisation of compacta $K$ which are basically embedded in $\mathbb{R}^{2}$ which can be formulated in terms of special sequences of points called arrays, using arguments from functional analysis. In this paper we give a simple topological proof of the implication: if there exists an array in $K$ of length $n$ for any $n \in \mathbb{N}$, then $K$ is not basically embedded.

\section{INTRODUCTION}

In his famous lecture at the International Congress of Mathematics in Paris in 1900, Hilbert formulated 23 problems which, in his opinion, were important for further development of mathematics. The thirteenth of these problems contained implicitly the conjecture that not all continuous functions of three variables are representable as superpositions of continuous functions of two variables. This conjecture was refuted in a series of papers by Arnold $[\mathbf{1}, \mathbf{3}]$ and Kolmogorov $[\mathbf{7}, \mathbf{6}]$.

Their result can be stated in the following way. A compactum $K \subset \mathbb{R}^{N}$ is said to be basically embedded in $\mathbb{R}^{N}$ if for each continuous function $f: K \rightarrow \mathbb{R}$ there exist continuous functions $g_{1}, \ldots, g_{N}: \mathbb{R} \rightarrow \mathbb{R}$ such that $f\left(x_{1}, \ldots, x_{N}\right)=g_{1}\left(x_{1}\right)+\cdots+g_{N}\left(x_{N}\right)$ for each point $\left(x_{1}, \ldots, x_{N}\right) \in K$. Kolmogorov [6] proved that the cube $\mathbb{I}^{n}$ can be basically embedded into $\mathbb{R}^{2 n+1}$. This result was generalised by Ostrand [9] who proved that every $n$-dimensional compact metric space is basically embeddable into $\mathbb{R}^{2 n+1}$. His result should be compared to the Nöbeling-Menger theorem [5] which says that every $n$-dimensional compact metric space $K$ is embeddable into $\mathbb{R}^{2 n+1}$. It is known that the number $2 n+1$ in this theorem can not be reduced, a famous counterexample was given by Van Kampen [15] and Flores [4] who showed that the $n$-skeleton of a $(2 n+2)$-simplex does not embed into $\mathbb{R}^{2 n}$. But there certainly exist $n$-dimensional compact metric spaces

Received 22nd May, 2003

The first author was supported in part by the Ministry for Education, Science and Sport of the Republic of Slovenia Research Program No. 101-509. This work was completed during a visit of the second author at the Faculty of Computer and Information Science of the University of Ljubljana which was financed thorugh grants from the CEEPUS project SK-47 and from the Slovak Grant Agency VEGA 1/7555/20.

Copyright Clearance Centre, Inc. Serial-fee code: 0004-9727/03 \$A2.00+0.00. 
which are embeddable into $\mathbb{R}^{2 n}$, for example, this is true for every $n$-dimensional manifold [14]. On the contrary, for basic embeddings Sternfeld [12] proved the following theorem.

THEOREM 1. [Sternfeld] For $n \geqslant 2$ an $n$-dimensional compact metric space $K$ is not basically embeddable into $\mathbb{R}^{2 n}$.

For $N \geqslant 3$, a compact metric space $K$ is thus basically embeddable into $\mathbb{R}^{N}$ if and only if $\operatorname{dim} K<N / 2$. Clearly $K$ is basically embeddable into $\mathbb{R}^{1}$ if and only if it is embeddable into $\mathbb{R}^{1}$. It remains to characterise compact metric spaces that are basically embeddable into the plane. This problem was first raised by Arnold [2] and the solution was given by Sternfeld [13], who gave a characterisation of compacta which are basically embedded into the plane, which was restated by Skopenkov [11] in the following way.

THEOREM 2. For a compactum $K \subset \mathbb{R}^{2}$ the following conditions are equivalent:

(A) Let an array be a sequence of points in $\mathbb{R}^{2}$ such that each consecutive pair bounds a vertical or a horizontal segment, and any two consecutive segments are orthogonal. Then $K$ does not contain any array of $n$ points for some $n$.

(B) The embedding $K \subset \mathbb{R}^{2}$ is basic.

(E) Let $p$ and $q$ denote the two projections, $p(x, y)=x$ and $q(x, y)=y$, let $|X|$ denote the cardinality of $X$, and let

$$
E(K)=\left\{(x, y) \in K|| p^{-1}(x) \cap K \mid \geqslant 2 \text { and }\left|q^{-1}(y) \cap K\right| \geqslant 2\right\} .
$$

Then $E^{n}(K)=E(E(\ldots E(K) \ldots))=\emptyset$ for some $n$.

Using Theorem 2, Skopenkov [11] gave a characterisation of finite graphs which are basically embeddable into the plane which resembles Kuratowski's condition for a graph to be planar: a finite graph is basically embeddable into the plane if and only if it does not contain a circle, a pentod, or a cross with branched ends. He also gave a characterisation of path-connected continua that are basically embeddable into the plane.

Despite these simple topological characterisations, the proof of Theorem 2 is not direct, but uses advanced arguments and tools from functional analysis. It would be therefore desirable to find a direct, topological proof, which would consequently also provide an elementary proof of Skopenkov's characterisation. In this paper we describe an elementary proof of the implication $(B) \Rightarrow(A)$ which is direct and much shorter than Sternfeld's original one. Our proof is by contradiction. For a compactum $K \subset \mathbb{R}^{2}$ which contains an array of length $n$ for any $n \in \mathbb{N}$ we explicitly construct a function $f: K \rightarrow \mathbb{R}$ which cannot be expressed as a sum $f(x, y)=g(x)+h(y)$, where $g, h: \mathbb{R} \rightarrow \mathbb{R}$ are continuous functions.

It is reasonable to extend the question of basically embedded sets from continuous functions to other categories of functions, for example $C^{n}$, smooth, Lipschitz, ... In the smooth cetagory, Repovš and Željko [10] proved that there exists a $K$ which is basically 
embedded in $\mathbb{R}^{2}$ and a $C^{\infty}$ function $f(x, y)$ such that for every decomposition $f(x, y)$ $=g(x)+h(y)$ the functions $g$ and $h$ are only $C^{1}$.

We would like to thank Arkadiy Skopenkov and Dušan Repovš for motivation for this paper. Our thanks to Arkadiy Skopenkov also go for the extensive conversations on the topic of this paper and for many helpful suggestions.

\section{Some examples and the Statement of the Result}

Let $C(X)$ denote the set of real valued continuous functions on $X$ with the supremum norm.

Definition 1: We say that a set $K \subset \mathbb{R}^{2}$ is basically embedded in $\mathbb{R}^{2}$ (or that the embedding $K \subset \mathbb{R}^{2}$ is basic) if for each function $f \in C(K)$ there exist functions $g, h \in C(\mathbb{R})$ such that for all $(x, y) \in K$ :

$$
f(x, y)=g(x)+h(y) .
$$

In this paper we shall consider compact sets, so we may assume without loss of generality that $K \subset \mathbb{I}^{2}$, where $\mathbb{I}:=[-1,1]$.

It is easy to see that any embedding of the one, two or three point set in $\mathbb{I}^{2}$ is basic. The following example shows that there exist embeddings of the four point set which are not basic.

EXAMPLE 1. Let $f$ be a continuous function on the four point set

$$
A=\left\{\left(x_{1}, y_{1}\right),\left(x_{2}, y_{2}\right),\left(x_{3}, y_{3}\right),\left(x_{4}, y_{4}\right)\right\}
$$

and assume that $f(x, y)=g(x)+h(y)$ for some $g, h \in C(\mathbb{I})$. Let $g\left(x_{i}\right)=: \alpha_{i}$ and $h\left(y_{i}\right)=: \beta_{i}, i=1, \ldots, 4$. The set $A$ is basically embedded if and only if the system of linear equations in the unknowns $\alpha_{i}, \beta_{i}$,

$$
\alpha_{i}+\beta_{i}=f\left(x_{i}, y_{i}\right), \quad i=1, \ldots, 4
$$

has a solution for every choice of values $f\left(x_{i}, y_{i}\right)$. If the four points in $A$ form a quadrilateral with sides parallel to the coordinate axes, so that for example $\alpha_{1}=\alpha_{2}, \alpha_{3}=\alpha_{4}$, $\beta_{1}=\beta_{4}$, and $\beta_{2}=\beta_{3}$, then the resulting system in the unknowns $\alpha_{1}, \alpha_{3}, \beta_{1}$ and $\beta_{2}$ is degenerate, and therefore does not have a solution for every choice of values $f\left(x_{i}, y_{i}\right)$, $i=1, \ldots, 4$. Thus, the four point set forming a quadrilateral with sides parallel to the axes is not basically embedded in $\mathbb{I}^{2}$.

Definition 2: A sequence of points $\left\{a_{i}\right\}_{i \in I}$ in $\mathbb{R}^{2}$, where $I=\{1, \ldots, n\}$ for some $n \in \mathbb{N}$ or $I=\mathbb{N}$, is called an array if each two consecutive points $a_{i}$ and $a_{i+1}$ are distinct, each segment $\left[a_{i}, a_{i+1}\right]$ is parallel to one of the coordinate axes, and each two consecutive segments $\left[a_{i-1}, a_{i}\right]$ and $\left[a_{i}, a_{i+1}\right]$ are orthogonal to each other. A finite array consisting of $n$ points such that $a_{1}=a_{n}$ is closed. 
EXAMPLE 2. The sequence $a_{1}:=\left(x_{1}, y_{1}\right), a_{2}:=\left(x_{2}, y_{1}\right), a_{3}:=\left(x_{2}, y_{2}\right), a_{4}:=\left(x_{1}, y_{2}\right)$, $a_{5}:=\left(x_{1}, y_{1}\right)$ is a closed array. As we have seen in Example 1 the set $\left\{a_{1}, a_{2}, a_{3}, a_{4}\right\}$ is not basically embedded in $\mathbb{R}^{2}$.

The main result of this paper is an elementary proof of the following theorem.

THEOREM 3. If a compact set $K \subset \mathbb{I}^{2}$ contains an array of $n$ points for every $n \in \mathbb{N}$ then the embedding $K \subset \mathbb{I}^{2}$ is not basic.

Every closed array can be periodically extended to an array of $n$ points for every $n \in \mathbb{N}$. We first consider this special case.

LEMmA 1. If a compact set $K \subset \mathbb{I}^{2}$ contains a closed array then the embedding $K \subset \mathbb{I}^{2}$ is not basic.

Proof: Let $K$ contain the closed array $A_{m}=\left\{a_{i}\right\}_{i=1}^{m}$. We may assume that all points except the first and the last are distinct, that is, $a_{i} \neq a_{j}$ for all $i \neq j,\{i, j\}$ $\neq\{1, m\}$. Let $\left[a_{1}, a_{2}\right]$ be parallel to the $x$ axis.

Let $f \in C(K)$ and let us assume that there are functions $g, h \in C(\mathbb{I})$ such that $f=g+h$. Since $A_{m}$ is an array, we obtain the following system of equations for the values $g\left(x_{i}\right)$ and $h\left(y_{i}\right)$, where $x_{i}$ and $y_{i}$ appear as coordinates of points of the array:

$$
f\left(a_{2 i-1}\right)=g\left(x_{i}\right)+h\left(y_{i}\right), \quad f\left(a_{2 i}\right)=g\left(x_{i+1}\right)+h\left(y_{i}\right), \quad i \leqslant(m+1) / 2 .
$$

Depending of whether $m=2 k+1$ is odd or $m=2 k$ is even, one of the following two possibilities occurs:

$$
\begin{aligned}
f\left(a_{2 k}\right) & =g\left(x_{1}\right)+h\left(y_{k}\right) \\
f\left(a_{2 k+1}\right) & =g\left(x_{1}\right)+h\left(y_{1}\right)
\end{aligned} \quad \text { or } \quad \begin{aligned}
f\left(a_{2 k-1}\right) & =g\left(x_{k}\right)+h\left(y_{1}\right) \\
f\left(a_{2 k}\right) & =g\left(x_{1}\right)+h\left(y_{1}\right) .
\end{aligned}
$$

In both cases,

$$
\sum_{i=1}^{m}(-1)^{i} f\left(a_{i}\right)=0 .
$$

Since there certainly exist functions in $C(K)$ that do not satisfy equation (1), $K$ is not basically embedded in $\mathbb{I}^{2}$.

EXAMPLE 3. It follows that an ellipse

$$
C_{1}:=\left\{(x, y) \in \mathbb{R}^{2} \mid \frac{(x-u)^{2}}{a^{2}}+\frac{(y-v)^{2}}{b^{2}}=r^{2}\right\}
$$

with axes parallel to the coordinate axes is not basically embedded in $\mathbb{R}^{2}$ since every point which is not a vertex lies on a closed array of length 5 .

EXAMPLE 4. We shall call the bipartite graph $K_{1,4}$ the $X$-graph. The following embedding of the $X$-graph:

$$
X_{1}:=\left\{(x, y) \in \mathbb{I}^{2} \mid x+y=0\right\} \cup\left\{(x, y) \in \mathbb{I}^{2} \mid x-y=0\right\}
$$


is not basic, since it contains a closed array. The following embedding of the $X$-graph

$$
X_{2}:=\left\{(x, 0) \in \mathbb{I}^{2}\right\} \cup\left\{(0, y) \in \mathbb{I}^{2}\right\}
$$

is basic, though. If $f \in C\left(X_{2}\right)$ then $f=g+h$ where

$$
\begin{array}{ll}
g(x):=f(x, 0)-f(0,0), & x \in \mathbb{I} \\
h(y):=f(0, y), & y \in \mathbb{I} .
\end{array}
$$

\section{Proof of Theorem 3}

It remains to consider compact sets containing arrays of any length, but no closed arrays. The following lemma represents a crucial step in the proof of this case.

LEMma 2. Assume that a compact set $K \subset \mathbb{I}^{2}$ contains no closed arrays, but contains an array of $m$ points for every $m \in \mathbb{N}$. Then there exists a sequence of functions $\{f\}_{n=3}^{\infty} \subset C(K)$ of norm 1 such that for each $n \geqslant 3$ the following property holds: if $f \in C(K)$ is such that $f=g+h$ for some $g, h \in C(\mathbb{I})$ and $\left\|f-f_{n}\right\|<1 / n$, then $\|g\|>n$.

PROOF: Since $K$ contains no closed arrays, the points of every array $A_{m}, m \in \mathbb{N}$, are all distinct.

To define the function $f_{n}$, we take an array $A_{2 n+4}=\left\{a_{i}\right\}_{i=1}^{2 n+4}$ of length $2 n+4$, put

$$
f_{n}\left(a_{i}\right):=(-1)^{i}, \quad i=1,2, \ldots, 2 n+4
$$

and extend $f_{n}$ continuously to $K$.

Let $f \in C(K)$ be such that $\left\|f-f_{n}\right\|<1 / n$. Then

$$
f\left(a_{i}\right)=(-1)^{i}\left(1+\delta_{i}\right), \quad \delta_{i} \in\left(-\frac{1}{n}, \frac{1}{n}\right)
$$

for all $i$. Assume that $f=g+h$ for some $g, h \in C(\mathbb{I})$. Without loss of generality let $a_{1}=\left(x_{1}, y_{1}\right), a_{2}=\left(x_{2}, y_{1}\right), a_{3}=\left(x_{2}, y_{2}\right)$, et cetera and let us denote $g\left(x_{1}\right)=: \gamma$. We consider the values of $g, h$ in the points $a_{i}$ of the array $A_{2 n+4}$.

For the point $a_{1}$ we have $f\left(x_{1}, y_{1}\right)=-1-\delta_{1}, g\left(x_{1}\right)=\gamma$ and $h\left(y_{1}\right)=f\left(x_{1}, y_{1}\right)$ $-g\left(x_{1}\right)=-1-\gamma-\delta_{1}$. For $a_{2}$ we have $f\left(x_{2}, y_{1}\right)=1+\delta_{2}, g\left(x_{2}\right)=f\left(x_{2}, y_{1}\right)-h\left(y_{1}\right)$ $=2+\gamma+\left(\delta_{1}+\delta_{2}\right)$ and $h\left(y_{1}\right)=-1-\gamma-\delta_{1}$. For $a_{2 n+4}$ we have $f\left(x_{n+3}, y_{n+2}\right)=1+\delta_{2 n+4}$, $g\left(x_{n+3}\right)=(2 n+4)+\gamma+\sum_{i=1}^{2 n+4} \delta_{i}$ and $h\left(y_{n+2}\right)=-(2 n+3)-\gamma-\sum_{i=1}^{2 n+3} \delta_{i}$.

If $|\gamma|>n$ then clearly $\left|g\left(x_{1}\right)\right|>n$. If $|\gamma| \leqslant n$ and $n \geqslant 3$ then

$$
\left|g\left(x_{n+3}\right)\right|=\left|2 n+4+\gamma+\sum_{i=1}^{2 n+4} \delta_{i}\right| \geqslant 2 n+4-n-\frac{2 n+4}{n}>n .
$$

So in both cases, $\|g\|>n$ for $n \geqslant 3$. 
Proof: [Proof of Theorem 3] The proof will be by contradiction. So let us suppose that for every $n \in \mathbb{N}$ there exists an array of length $n$ in $K$ and the embedding $K \subset \mathbb{I}^{2}$ is basic. Since $K$ is basically embedded there are no closed arrays by Lemma 1 . By Lemma 2, there exists a sequence of functions $\left\{f_{n}\right\}_{n=1}^{\infty} \subset C(K)$ of norm 1 such that for each $n \geqslant 3$ the following is true: if $f \in C(K)$ is of the form $f=g+h$ for some $g, h \in C(\mathbb{I})$ and it has the property that $\left\|f-f_{n}\right\|<1 / n$, then the norm of $g$ is greater than $n$.

The idea of the proof is the following. We shall construct a sequence of numbers $\left\{\lambda_{n}\right\}_{n=1}^{\infty}$ from $(0,1]$ and an ascending sequence of natural numbers $\left\{s_{n}\right\}_{n=1}^{\infty}$ such that the series

$$
\sum_{i=1}^{\infty} \lambda_{i} f_{s_{i}}
$$

is convergent in $C(K)$ but its sum $F \in C(K)$ is not expressible in the form $F=G+H$ for any $G, H \in C(\mathbb{I})$. Denote the partial sums of the series (2) by

$$
F_{n}:=\sum_{i=1}^{n} \lambda_{i} f_{s_{i}}
$$

The construction of the sequences $\left\{\lambda_{n}\right\}_{n=1}^{\infty}$ and $\left\{s_{n}\right\}_{n=1}^{\infty}$ is by induction. Let $\lambda_{1}:=1$ and $s_{1}:=3$. Assuming that $\lambda_{n-1}$ and $s_{n-1}$ have already been constructed, let

$$
\lambda_{n}:=\frac{\lambda_{n-1}}{2\left(s_{n-1}\right)^{2}} \text {. }
$$

Because the set $K$ is basically embedded, there exists a representation of the partial sum

$$
F_{n-1}=G_{n-1}+H_{n-1}, \quad G_{n-1}, H_{n-1} \in C(\mathbb{I}) .
$$

Let $s_{n}$ be a any number in $\mathbb{N}$ such that $s_{n}>s_{n-1}$ and

$$
\lambda_{n} s_{n}>\left\|G_{n-1}\right\|+n
$$

Let us consider the series $\sum_{i=1}^{\infty} \lambda_{i} f_{s_{i}}$ we have constructed. Since $s_{n} \geqslant n+1$, it follows by (3) that

$$
\lambda_{n} \leqslant \frac{1}{n^{2}}
$$

for all $n \in \mathbb{N}$. Therefore,

$$
\sum_{i=1}^{\infty}\left|\lambda_{i}\right|\left\|f_{s_{i}}\right\|=\sum_{i=1}^{\infty} \lambda_{i} \leqslant \sum_{i=1}^{\infty} \frac{1}{i^{2}}
$$

so the series converges in $C(K)$ to its sum

$$
F:=\sum_{i=1}^{\infty} \lambda_{i} f_{s_{i}} .
$$


For each $n \in \mathbb{N}$, we have

$$
\begin{aligned}
\left\|F-F_{n}\right\| & =\left\|\sum_{i=n+1}^{\infty} \lambda_{i} f_{s_{i}}\right\| \leqslant \sum_{i=n+1}^{\infty}\left|\lambda_{i}\right|\left\|f_{s_{i}}\right\|=\sum_{i=n+1}^{\infty} \lambda_{i} \\
& =\lambda_{n+1} \sum_{j=0}^{\infty} \frac{1}{2^{j}\left(s_{n+1}\right)^{2}\left(s_{n+2}\right)^{2} \cdots\left(s_{n+j}\right)^{2}}<\lambda_{n+1} \sum_{j=0}^{\infty} \frac{1}{2^{j}}=2 \lambda_{n+1}
\end{aligned}
$$

so

$$
\left\|F-F_{n}\right\|<2 \lambda_{n+1} .
$$

Let $F=G+H$ for some $G, H \in C(\mathbb{I})$ and let $n \in \mathbb{N}$. Then

$$
\left\|F-F_{n}\right\|=\left\|F-F_{n-1}-\lambda_{n} f_{s_{n}}\right\|=\lambda_{n}\left\|\frac{F-F_{n-1}}{\lambda_{n}}-f_{s_{n}}\right\| .
$$

Let us denote

$$
f:=\frac{F-F_{n-1}}{\lambda_{n}}
$$

By (5),

$$
\left\|f-f_{s_{n}}\right\|<\frac{2 \lambda_{n+1}}{\lambda_{n}}=\frac{1}{\left(s_{n}\right)^{2}}<\frac{1}{s_{n}} .
$$

By Lemma 2, this implies that for any representation $f=g+h$ we have $\|g\|>s_{n}$. Since

$$
f=\frac{F-F_{n-1}}{\lambda_{n}}=\frac{G-G_{n-1}}{\lambda_{n}}+\frac{H-H_{n-1}}{\lambda_{n}}
$$

is such a representation, it follows that

$$
\left\|\frac{G-G_{n-1}}{\lambda_{n}}\right\|>s_{n}
$$

Then by (4),

$$
\|G\|+\left\|G_{n-1}\right\| \geqslant\left\|G-G_{n-1}\right\|>\lambda_{n} s_{n}>\left\|G_{n-1}\right\|+n,
$$

and therefore

$$
\|G\|>n \text {. }
$$

This implies that the norm $\|G\|$ is greater than $n$ for all $n \in \mathbb{N}$, which is not possible.

According to Skopenkov [11] one of the forbidden subgraphs in a graph that is basically embeddable into the plane is the circle. We conclude this paper with an elementary proof of this fact using Theorem 3. First let us look at two special cases.

An array $A_{m} \subset K$ of finite length $m$ which cannot be extended to a longer array by adding points at the beginning or at the end will be called a maximal array. A closed array of $n$ points such that the beginning and ending segments are orthogonal can be extended to an array of arbitrary length, so it is not contained in a maximal array. 
EXAMPLE 5. Let $C_{2} \subset \mathbb{I}^{2}$ be an ellipse with axes rotated with respect to the coordinate axes:

$$
C_{2}:=\left\{(x, y) \mid a x^{2}+b x y+c y^{2}=1\right\}, \quad a c-b^{2}>0, a+c>0 .
$$

Let $A_{m}=\left\{a_{i}\right\}_{i=1}^{m}$ be an array of length $m$ in $C_{2}$ ending with a horizontal segment, and let $a_{m}=\left(x_{m}, y_{m}\right)$. Clearly $y_{m}$ cannot be an extreme value of the projection to the $y$ axes $q\left(C_{2}\right)$, since this would imply that the horizontal line through $a_{m}$ intersects $C_{2}$ only in this one point, contradicting the fact that $a_{m-1}$ is a different point in $C_{2}$ on the same horizontal line. If $x_{m}$ is not an extreme value of $p\left(C_{2}\right)$, then the vertical line through $a_{m}$ intersects $C_{2}$ in a second point which we denote by $a_{m+1}=\left(x_{m+1}, y_{m+1}\right)$, where $x_{m+1}=x_{m}$ (which can possibly coincide with some $a_{i}, i<(m-1)$ ). Thus $A_{m}$ can be extended to an array of length $m+1$. A similar argument can be used if the array $A_{m}$ ends with a vertical segment. Given an array $A_{m}$ ending in one of the extreme points, the opposite array $\bar{A}_{m}$ with $\bar{a}_{0}=a_{m}, \ldots, \bar{a}_{m}=a_{0}$ can also be extended until it possibly reaches an extreme point with respect to one of the coordinates. This shows that all maximal arrays begin and end in one of the four extreme points of the ellipse.

Since vertical and horizontal lines intersect $C_{2}$ in at most two points, there is only one maximal array starting in any extreme point, so $C_{2}$ contains at most two maximal arrays each connecting two of the four extreme points. Since these two arrays contain at most finitely many points, there must exist a point on the ellipse that is not in any one of these two arrays. Any array starting at this point can thus be extended to an array of arbitrary length. By Theorem 3 , the ellipse $C_{2}$ is therefore not basically embedded in the plane.

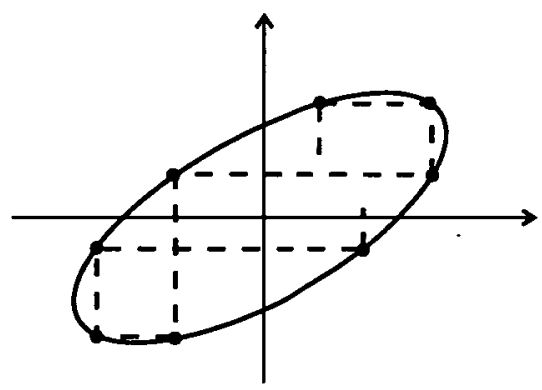

Figure 1: An infinite array in a slanted ellipse

The argument used in the Example 5 does not work in general, as the following example shows.

EXAMPLE 6. Let $T$ be the triangle with one horizontal and one vertical side. Then every point on $T$ lies on a maximal array of length at most 5 , as Figure 2 shows.

Corollary 1. A simple closed curve $C$ is not basically embedded in the plane. 


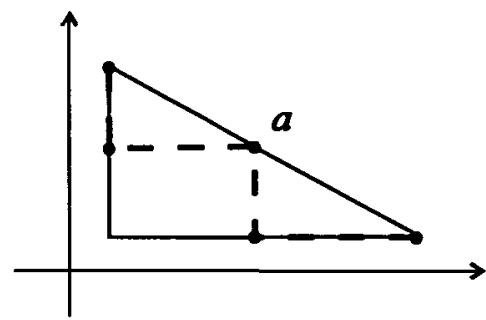

Figure 2: An array of length 5 containing the point $a$

Proof: The characteristic property of simple closed curves that we use is that every horizontal or vertical line through a point which is not an extreme point with respect to the $y$ or $x$ coordinate respectively, intersects $C$ in at least two points. If $a$ is an end point of a maximal array in $C$, then $a$ must be extreme with respect to one of the coordinates, for example with respect to $x$. Let us assume that $a$ is not extreme with respect to $y$. If there exists a point $b$ on $C$, different from $a$ such that $p(b)=p(a)$, then an array ending in $a$ can either be extended by adding $b$ (if the final segment is horizontal) or by adding a point from $C$ on the horizontal line through $a$ (if the final segment is vertical). Thus, all maximal arrays in $C$ begin and end in points which are both extreme with respect to the $y$ or $x$ coordinate, and such that the horizontal or vertical line, respectively, through these points has no other intersections with $C$. There are at most four such points on $C$.

If there exists a point on $C$ which is not an element of a maximal array of finite length, then $C$ is not basically embedded by Theorem 3 . Assume therefore, that every point on $C$ is an element of a maximal array of finite length. Since there are infinitely many points on $C$ and every such array contains only finitely many points, there must be infinitely many such arrays. Since there are only four possible beginning and ending points, infinitely many of these arrays must begin in the same one of these points which we denote by $c$ and also end in the same one of the remaining three points which we denote by $d$. Let $A=\left\{a_{i}\right\}_{i-1}^{n}$ and $B=\left\{b_{j}\right\}_{i=1}^{m}$ be two of these arrays, and let $a_{i_{k}}$ and $b_{j_{t}}$ be the last points of $A$ and $B$ that are different. Then one of the coordinates of the points $a_{i_{k}}$ and $b_{j_{l}}$ must be the same, so the union $\left\{a_{i}\right\}_{i=0}^{i_{k}} \cup\left\{b_{j_{l}-j}\right\}_{j=0}^{j_{l}}$ is an array, beginning and ending in the point $c$. By Lemma $1, C$ is not basically embedded.

\section{REFERENCES}

[1] V.I. Arnold, 'On functions of three variables', (in Russian), Dokl. Akad. Nauk. 114 (1957), 679-681.

[2] V.I. Arnold, 'Problem 6', Math. Ed. 3 (1958), 273-273.

[3] V.I. Arnold, 'Representation of continuous functions of three variables by the superpositions of continuous functions of two variables', (in Russian), Math. Sb. 48 (1959), 3-74. 
[4] A. Flores, 'Über $m$-dimensionale Komplexe, die im $R_{2 m+1}$ absolut selbsverschlungen sind', Erg. Math. Kolloqu. 6 (1935), 4-6.

[5] W. Hurewicz and H. Wallman, Dimension theory (Princetion University Press, Princeton, N.J., 1941).

[6] A.N. Kolmogorov, 'On the representations of continuous functions of many variables by superpositions of continuous functions of one variable and addition', (in Russian), Dokl. Akad. Nauk. 114 (1957), 953-956.

[7] A.N. Kolmogorov, 'On the representations of continuous functions of many variables by superpositions of continuous functions fewer variables', (in Russian), Dokl. Akad. Nauk 108 (1956), 179-182.

[8] M. Levin, 'Dimension and superposition of continuous functions', Israel J. Math. 70 (1990), 205-218.

[9] P.A. Ostrand, 'Dimension of metric spaces and Hilbert's problem 13', Bull. Amer. Math. Soc. 71 (1965), 619-622.

[10] D. Repovš and M. Željko, 'On basic embeddings into the plane', (Preprint).

[11] A. Skopenkov, 'A description of continua basically embeddable in $R^{2}$ ', Topology Appl. 65 (1995), 29-48.

[12] Y. Sternfeld, 'Dimension, superposition of functions and separation of points, in compact metric spaces', Israel J. Math. 50 (1985), 13-52.

[13] Y. Sternfeld, 'Hilbert's 13th problem and dimension', Lecture Notes in Math. 1376 (1989), 1-49.

[14] H. Whitney, 'The self-intersections of a smooth $n$-manifold in $2 n$-space', Ann. of Math. (2) 45 (1944), 220-246.

[15] E.R. van Kampen, 'Komplexe in euklidischen Räumen', Abh. Math. Sem. Univ. Hamburg 9 (1932), 72-78, 152-153.

Institute of Mathematics

Physics and Mechanics

University of Ljubljana

Slovenia

e-mail: neza.mramor-kosta@fri.uni-lj.si
Faculty of Science

P. J. Šafárik University Košice

Slovakia

e-mail: etrenkle@cs.science.upjs.sk 